\title{
Literarische Gestaltung des Grafen Albert Joseph Hoditz (1706-1778) in der Novelle August Lewalds "Roswalde"
}

\author{
Literary Design of Count Albert Joseph Hoditz (1706-1778) \\ in the Novella by August Lewald "Roswalde"
}

Iveta Zlá

\begin{abstract}
The article focuses on the literary design of Count Albert Joseph Hoditz (1706-1778) in the novella by August Lewald "Roswalde", which was dominant marked by the influence of the literature of Biedermeir. Since the literary composition of that nobleman has been paid in the research literature almost no attention, the present article is to be understood as an innovative contribution to the Hoditz research in the literary historical intentions.
\end{abstract}

\section{Keywords}

Count Albert Joseph Hoditz, literary designs of Count Albert Joseph Hoditz, its role in the history of European literature, Biedermeier, literary creation by August Lewald 


\section{Einführung in die Thematik}

August Lewald (1792-1871) ist in die deutschsprachige Literatur nicht nur als ein Autor und Publizist eingegangen, sondern seine Biographie weist auch auf seine Nähe zur schauspielerischen Tätigkeit hin. Das literarische Schaffen Lewalds wurde durch die künstlerischen Impulse der Romantik sowie des Biedermeiers und Jungen Deutschlands geprägt und trägt teilweise autobiographische Züge. In seine Novellen und Romane haben historische Ereignisse und Persönlichkeiten Eingang gefunden und mündeten in die Darstellung eines Zeit- und Sittengemäldes. ${ }^{1}$

Das kulturelle Engagement des Grafen Albert Joseph Hoditz (1706-1778) wurde in seinem Rosswalder Dominium umgesetzt, dessen Garten durch die Verwirklichung der landesarchitektonischen Ambitionen dieses Adligen zum „Reich der Musen und Grazien“ verwandelt wurde. Die als „Schlesisches Versailles“ berühmte Gegend wurde nicht nur durch die Dramen-, Opern- und Ballettaufführungen gekennzeichnet, sondern sie wurde auch zum Mittelpunkt zahlreicher Festivitäten. Diese märchenhafte Landschaft, der Landessitz des Grafen Hoditz sowie sein zwischen Genialität und Wahnsinn schwankendes Naturell haben eine vielfältige Resonanz in der europäischen Literatur gefunden. Einen Einblick in das Rosswalder kulturelle Panorama und in die Lebensweise des Grafen Albert Joseph Hoditz sowie in das durch die Atmosphäre zwischen Barock und Aufklärung geprägte historisch-kulturelle Klima bietet im literarischen Gewande die Novelle August Lewalds „Roswalde“. Sie ist 1833 in der Sammlung „Novellen“2 erschienen und bleibt thematisch dem Biedermeier treu. Neben der Flucht in die idyllische Gartenlandschaft und in die kulturelle Atmosphäre der Rosswalder Schlossresidenz sowie dem Einblick in die künstlerischen Aktivitäten des Grafen Hoditz stehen die moralischfamiliären Werte wie Ehe, Treue und Tugend im Vordergrund der Novellenhandlung.

Die vorliegende Untersuchung setzt sich zum Ziel, das literarische Bild des Grafen Hoditz und seines Rosswalder Dominiums in der Novelle „Roswalde“ August Lewalds zu beleuchten und die literaturhistorischen Facetten seiner Gestaltung darzulegen. Die literarische Erfassung des Grafen Hoditz wird vor dem Hintergrund seiner Beziehung zu Signora Doristea sowie zu Friedrich II. vorgestellt, um auf sein kulturelles Engagement hinzuweisen. Darüber hinaus ist die Studie mit der Intention verbunden, die gesellschaftlich-kulturelle Atmosphäre im Rosswalder Dominium zu skizzieren. Die Auseinandersetzung mit der literarischen Gestaltung des Grafen Hoditz geht von der Analyse der Berührungspunkte zwischen der literarischen Figur dieses Adligen und seinem realen Vorbild aus und zielt auf die Betrachtung dieser literarischen Gestalt als einer Schlüsselfigur ab. Da den literarischen Gestaltungen dieses Adligen bisher in der Forschungsliteratur fast keine Aufmerksamkeit geschenkt wurde, ist der vorliegende Artikel als innovativer Beitrag für die Hoditz-Forschung in den literaturhistorischen Intentionen zu verstehen.

1 Vgl. Selbmann, Rolf: Lewald, August. In: Neue Deutsche Biographie. Bd. 14. München 1985, S. 408 f.

2 Vgl. Lewald, August: Novellen. 3. Teil. Hamburg 1833. 


\section{Der Graf Albert Joseph Hoditz als Schlüsselfigur in der Novelle August Lewalds „Roswalde"}

Die in der Novelle als ein „Privatmann“ und folglich als „Graf Hoditz“ bezeichnete literarische Gestalt ist als eine Schlüsselfigur zu betrachten. Dies belegen zahlreiche Berührungspunkte der analysierten Novelle mit den historisch relevanten Informationen und die explizit thematisierte Absicht des Autors, den Grafen Hoditz in seiner Novelle literarisch zu gestalten. Die Andeutung dieser literarisch-historischen Parallelen und Unterschiede verfolgt das Ziel, auf die literaturwissenschaftlichen und -historischen Merkmale der Novelle hinzuweisen, durch die gleichsam die Fabulierungskunst Lewalds berührt wird. Diese Vorgehensweise gibt jedoch die Intention auf, zur historischen Untersuchung der Gemeinsamkeiten und Differenzen zwischen dieser Novelle und ihrem historischen Hintergrund zu werden.

Bereits die einleitenden Worte der Novelle deuten die geographische Lage des Rosswalder Dominiums und sein kulturelles Renommee an, das mit Mitte des 18. Jh. verbunden ist. Obwohl an der untersuchten Textstelle der Graf Hoditz explizit nicht genannt wird, ist die Übereinstimmung der Novellenfigur mit ihrem historischen Vorbild kontextuell zu belegen: „In dem fruchtreichen Lande, unweit des erzbischöflichen Sitzes von Ollmütz, erhob sich Mitte des vorigen Jahrhunderts aus seinen künstlich angelegten Parks, springenden Wassern und seiner marmornern Götterversammlung das wunderbare Roßwalde, welches die prachtliebende Eitelkeit ihres Privatmannes, im kühnen Wetteifer, zu einem deutschen Versailles erheben wollte. “3 Das Bild des Grafen Hoditz in der untersuchten Textpassage sowie der Umriss der geographischen Lage des Rosswalder Herrschaftsguts und nicht zuletzt die Abgrenzung des in der Novelle thematisierten Zeithorizonts gehen von den historisch-geographischen Informationen über das Rosswalder Dominium aus. Diese Übereinstimmung korrespondiert mit der aufgeworfenen, auf die literarische Gestaltung des Grafen Hoditz eingehenden These und stellt unter Beweis, dass August Lewald mit den Informationen über das Rosswalder kulturelle Panorama gut vertraut war.

Auf den Angaben aus der historisch ausgerichteten Forschungsliteratur ${ }^{4}$ bzw. aus dem Archivnachlass des Großgrundbesitzes Rosswald ${ }^{5}$ basiert ebenfalls die Auseinandersetzung mit der Beziehung des Grafen Albert Joseph Hoditz zur Markgräfin Wilhelmine Friederike Sophie von Sachsen-Weißenfels sowie mit den Kavalierreisen dieses Adligen. Dementsprechend wird das zweite Kapitel der untersuchten Novelle durch die folgenden Worte gekennzeichnet: „Graf Hoditz, der Schöpfer dieses Feensitzes, ein durch Reisen gebildeter Cavalier, lebte hier mit seiner Gemahlin Sophia, Witwe des Markgrafen Georg Wilhelm von Bayereuth [...].“6

3 Ebenda, S. 9.

4 Vgl. Myška, Milan: Hrabě Hodic a jeho svět. Zámecká kultura ve Slezsku mezi barokem a osvícenstvím. Ostrava 2011, S. 73-81.

5 Vgl. ZAO, FSR, Inventarnr.: 10452, Signatur: A 26.

6 Lewald, August: Novellen. 3. Teil. Hamburg 1833, S. 10. 
Obwohl der in das Jahr 1770 fallende Besuch des preußischen Monarchen in Rosswald in der Novelle nicht zeitlich näher bestimmt wurde, wird der Aufenthalt dieses Königs im „Schlesischen Versailles“ zu einem der Schwerpunkte der Novelle. Durch die vorliegende Thematisierung der Rosswalder Reise Friedrichs II. und Vorbereitung des für ihn veranstalteten Festes wird eine der Handlungslinien der Novelle eröffnet: „Der Graf erwartete einen Gast. Friedrich war's, der bei ihm einkehren wollte und alles wurde aufgeboten zu den reichen vorhandenen Kräften gesellten sich neue, weither verschiedene und sollte ein Kranz von Festlichkeiten werden, würdig des ruhmgekrönten Helden, zu dessen Ehren sie gefeiert wurden. "7 Obwohl die Bezeichnung des preußischen Königs unter dem historischen Aspekt als vage zu betrachten ist, taucht die Textstelle im literarischen Kontext auf, durch das die Übereinstimmung der literarischen Figur mit ihrem historischen Vorbild evident ist. ${ }^{8}$ Die in die Novelle eingegliederten Dialoge Friedrich II. mit dem Grafen Hoditz, seinen Bedienten, Signora Doristea und mit Voltaire sowie die Rolle des preußischen Monarchen bei der Trauung Doristeas mit dem Grafen von G., der darauffolgenden Scheidung und der Taufe ihres Sohns dürfen sich auf die Fabulierungskunst August Lewalds gestützt haben.

Der historischen Wirklichkeit entspricht ebenfalls das Gedicht des preußischen Königs, das Friedrich II. dem Grafen Hoditz dediziert hat. ${ }^{9}$ Diese Epistel fand in der Novelle August Lewalds die folgende Resonanz: „Friedrich war mit der Aufmerksamkeit seines Wirthes so zufrieden, daß er sein feenhaftes Roßwalde in einem eigenen Gedichte besang [...]. "10 Während die Eigenschaften, Lebensweise und Gastfreundschaft des Grafen Hoditz in der Novelle Lewalds einseitig positiv betrachtet werden, wird dieser Adlige in einigen historisch ausgerichteten Untersuchungen dieser Thematik als ein Sonderling bezeichnet. $^{11}$

Obwohl der Schlüsselfigur des Grafen Hoditz die Eigenschaften wie Freigiebigkeit und Großzügigkeit verliehen werden, wird in der analysierten Novelle Lewalds seine Einstellung zu finanziellen Angelegenheiten nicht thematisiert.

Das literarische Bild des Grafen Hoditz ist durch eine ganze Reihe der Berührungspunkte dieser Schlüsselfigur mit ihrem realen Vorbild gekennzeichnet. Die literarische Darstellung der mit dem Rosswalder Dominium verbundenen gesellschaftlich-kulturellen Vielfalt und die Hervorhebung der Rolle des Grafen Hoditz in der europäischen Literatur- und Kunstgeschichte unterstreicht auch hundert Jahre nach dem Tode dieses Adligen die Phänomenalität seines „Sitzes der Mus en und Grazien“ und seine Attraktivität für die künstlerische Erfassung.

7 Ebenda, S. 11.

8 Vgl. ebenda, S. $11 \mathrm{f}$.

9 Vgl. Friedrich II.: Euvres de Frédéric le Grand. Bd. XIII. Berlin 1849, S. 68-73.

10 Lewald, August: Novellen. 3. Teil. Hamburg 1833, S. 93 f.

11 Vgl. Trampler, Richard: Der Sonderling Hoditz in Rosswald. Eine historische Skizze. In: Österreichischschlesisches Vereinskalender für das Jahr 1877, Jg. 2. Wien 1876, S. 38-74. 


\section{3. "Graf Hoditz, der Schöpfer dieses Feensitzes". Das literarische Bild des Grafen Albert Joseph Hoditz in der Novelle „Roswalde”}

Obwohl die Novelle August Lewalds „Roswalde“ der gattungsspezifischen Singularität verpflichtet ist, durchziehen ihre Handlung einige thematische Akzente. Sie sind mit dem Rosswalder Herrschaftsgut und dessen Umgebung verbunden und verdeutlichen die Kontakte, die Denk- und Lebensweise des Grafen Hoditz sowie die gesellschaftlichkulturelle Atmosphäre im Rosswalder Dominium.

Für die Novelle ist die Überschneidung einiger Zeitlinien kennzeichnend, durch die die Rahmenhandlung markiert wird. Zum zeitlichen Ausgangspunkt wird die Atmosphäre im Rosswalder Herrschaftsgut am Vorabend des Besuchs Friedrichs II., deren Schilderung durch das Ankommen Doristeas, der ersten Sängerin Italiens, unterbrochen wird. Retrospektiv werden die Darlegung der Ausbildungs- und der Kavalierreisen des Grafen Hoditz sowie der Einblick in die künstlerische Laufbahn Doristeas geprägt. Zur Thematisierung des künftigen Geschehens avanciert das Bild des Rosswalder Aufenthalts Friedrichs II. sowie die Darlegung der Rolle Doristeas im mit dem Besuch des preußischen Monarchen verbundenen kulturellen Mosaik. Die Darstellung der Handlung lässt sich von der Zeitraffung nicht trennen, durch die ein Spannungsfeld zwischen den skizzierten Zeitlinien hergestellt wird.

Der Erzählmodus der Novelle ist als auktorial zu betrachten. Die Narration ist monologisch sowie dialogisch geprägt und der Erzählstandort ist durch die zeitliche Entfernung markiert. Die Frequenz des Erzählens ist als repetitiv zu bezeichnen, denn einige Ereignisse wurden von verschiedenen Blickpunkten her wiederholt erzählt.

Die Novelle wird vom Zitat aus der Korrespondenz Friedrichs II. eingeleitet, durch das eines ihrer inhaltlichen Schwerpunkte angedeutet wird. Die Kontakte des Grafen Hoditz zum preußischen Monarchen werden in der Novelle umrissen, dennoch spielt ihre Darstellung keine zentrale Rolle. Der Graf Hoditz wird im Fokus seiner Kreativität vorgestellt, durch die sowie durch sein Organisationstalent das kulturelle Programm des für den preußischen Monarchen vorbereiteten Festes mitgestaltet wird. Das künstlerische Arrangement der Feier überschattet in der Novelle die Darstellung der Kontakte dieses Adligen zum preußischen König.

An die Hervorhebung der künstlerischen Aktivitäten des Grafen Hoditz und die Vorbereitung des Festes wird die Schilderung der Lebensgeschichte der später als „Signora Doristea"12 berühmten Sängerin angelehnt, deren musikalisches Talent dank der Unterstützung des Grafen Albert Joseph Hoditz entwickelt wurde. Obwohl die Skizzierung der künstlerischen Karriere Doristeas in die Akzentuierung der moralischen und familiären Werte des Bürgertums mündet, zeichnet ihr künstlerischer Aufstieg gleichsam die Konturen des Rosswalder kulturellen Avancements. Das literarische Bild dieser Sängerin sowie des Rosswalder kulturellen Mosaiks wird durch einige Wendepunkte geprägt, die

12 Lewald, August: Novellen. 3. Teil. Hamburg 1833, S. 14. 
der Novellenhandlung Dynamik verleihen. ${ }^{13}$ Darüber hinaus werden vor ihrem Background auch einige Facetten der literarischen Verkörperung von Hoditz deutlich.

Signora Doristea ist als eine der zentralen Figuren der Novelle zu betrachten, die in die auktoriale Erzählsituation eingebettet ist. Ihre Lebensgeschichte und ihre von Erfolgen gekrönte musikalische Laufbahn werden durch die moralischen Vorstellungen der Aufklärung sowie durch die kulturellen Bestrebungen des Grafen Hoditz beeinflusst. Ihre musikalische Begabung wurde von diesem Adligen nicht nur entdeckt, sondern dank seiner finanziellen Unterstützung auch weiter entwickelt. Der retrospektive Einblick in die künstlerische Entwicklung Doristeas weist auf die kulturellen sowie erzieherischen Ambitionen des Grafen Albert Joseph Hoditz hin. Dieser Adlige wird im angeführten Kontext als derjenige vorgestellt, „der jedes Talent, wo er es entdeckte, aufs Eifrigste ans Licht zu ziehen bemüht war [...]. Der Graf fühlte sich beglückt, auf seinem Boden eine solche Wunderpflanze entkeimen zu sehen"14 und ließ Doris bei den größten Tanz- und Musikmeistern unterrichten. Die Bemühungen dieses Adligen um die Durchsetzung der

13 Bereits das Ankommen Doristeas wickelt sich nicht linear ab und wird durch das Verschwinden dieser Künstlerin gekennzeichnet. Das Sujet wird an dieser Textstelle durch die Figur des Schulmeisters Martin Heller entwickelt, durch dessen Gestaltung nicht nur die künstlerische Karriere Doristeas verdeutlicht wird, sondern auch die Mentalität und Moralvorstellungen des Bürgertums an der Schwelle zwischen Barock und Aufklärung berührt werden. Diese Lehrerfigur wird in das kommende zugunsten des preußischen Monarchen veranstaltete Fest als Autor des Lobgedichts zur Begrüßung des Königs eingebettet. Er versinkt bei dem Verfassen dieser Lobrede in die Erinnerungen an seine Tochter Doris, die von ihrem Kinderbild angeregt werden. Die Erinnerungen Marin Hellers an seine Tochter werden durch emotive Regungen erfüllt, die in seinen kurzen Monolog münden. Die Beschreibung des Bildes rückt die lange zeitliche Entfernung von der Tochter in den Vordergrund, deren Abbildung in die Kindheitsjahre reicht. Seinem Monolog hört Doristea $\mathrm{zu}$, die ihre Bedienten verlassen hat und vom Grafen Hoditz gesucht wird. Die literarische Gestaltung Martin Hellers sowie Doristeas wird durch emotive Vorgänge markiert, durch die ihre Vater-Tochter-Beziehung verdeutlicht und folglich vom auktorialen Erzähler explizit artikuliert wird. Der Gesang Signora Doristeas wurde als kultureller Gipfel des für Friedrich II. veranstalteten Festes gedacht. Die künstlerische Entwicklung von Doris war mit zahlreichen Erfolgen verbunden, auf die ihr Gesangstudium in Italien folgte. Vor dem Hintergrund des Aufstiegs von Doris lässt sich jedoch der Verlust ihrer Heimat nicht trennen. Ihre Abreise nach Italien wird mit den Worten des Schulmeisters Heller skizziert, die gleichsam die mit der Aufklärung verknüpften Moralgrundsätze umkreisen. An diese Textstelle knüpft eine Zeitraffung an, die ein halbes Jahr umschließt. Durch diese Ellipse wird das Gespräch des Grafen Hoditz mit Martin Heller eingeleitet, in dem dieser Adelige über die Erfolge Doristeas berichtet. Obwohl die erfolgreiche Laufbahn der Sängerin zahlreiche Möglichkeiten bot, hielt sich Doristea an die von ihren Eltern beigebrachten moralischen Prinzipien. Sie hat diese Grundsätze auch nach der Heirat mit dem Grafen von G. nicht verlassen, der sie bei ihrem Rosswalder Aufenthalt begleitete. Einen Wendepunkt stellt in der Novellenhandlung sein Anliegen an Doristea, während des für den preußischen Monarchen veranstalteten Festes nicht zu singen. Stattdessen wird sie in einem nahe liegenden Jagdschloss versteckt, damit sie nicht durchschaut, dass sie der Graf von G. betrogen hat. Die Heirat mit Doristea war inszeniert und der intrigante Adlige bemüht sich, die Hand der Marquise von Hauteroche zu gewinnen. Seine List wird jedoch dank der Initiative des Zauberkünstlers Philadelphia und später des preußischen Königs entdeckt. Obwohl Doristea ein Kind bekommt, ohne verheiratet zu sein, nehmen sie ihre Eltern auch mit dem Kind auf. Damit ihre Ehre unbefleckt bleibt, zwingt der preußische König den Grafen von G., die Sängerin zu heiraten und folglich unter ihrer Bewilligung eine offizielle Trennung zu vollziehen. Durch diese Akte enthält Doristea zugleich den Adelstitel und Friedrich II. wird selbst zum Taufpatten ihres Kindes. Obwohl der preußische König nicht mit dem Gesang Doristeas beglückt wurde, rettete er durch seine Initiative die Ehre Doristeas sowie das moralische Renommee des Adels.

14 Lewald, August: Novellen. 3. Teil. Hamburg 1833, S. 23. 
talentierten Künstler werden in der Novelle sowohl metaphorisch ${ }^{15}$ dargestellt, als auch durch die adverbialen Superlative ${ }^{16}$ in den Vordergrund gerückt.

Doris Heller erhielt in Rosswald nicht nur die Tanz- und Musikausbildung von Mr. La Beau und Mr. Rasioli ${ }^{17}$, sondern auch ihre Sitten erfuhren Vervollkommnung: „Sie mußte das Schulhaus verlassen und erhielt ihre Wohnung auf dem Schloße selbst, um im Umgange mit den Künstlern und vornehmen Leuten, die zu jeder Zeit dort versammelt waren, ihre Sitten abzuschleifen." ${ }^{18}$ Obwohl der Graf Hoditz mit der künstlerischen Vervollkommnung Doris Hellers die in Rosswald tätigen Tanz- und Musikmeister betraute, interessierte er sich auch weiter für die Fortschritte der künftigen erfolgreichen Sängerin. ${ }^{19}$ Ihre in Rosswald erworbene musikalische Ausbildung wurde in Italien weiter entwickelt und Doris wurde zur bewunderten Sängerin: „In Doris sollte die Welt bald eines der ersten Talente anstaunen. Es bedurfte nur einer leisen Anregung bei dem Grafen und die Tochter seines Schulmeisters wurde zu ihrer fernern Kunstausbildung nach Italien gesandt. " ${ }^{20}$

Der Aufenthalt Doristeas in Italien wird durch das zeitraffende Erzählen angedeutet, durch das sie in die Atmosphäre des für den preußischen Monarchen vorbereiteten Festes eingebettet wird. Sie wird von Hoditz für den künstlerischen Gipfel des Programms gehalten, der gleichsam die Bemühungen dieses Adligen um die Verbreitung der Kultur und Ausbildung repräsentierte.

Der Graf Hoditz wird nicht nur vor dem Hintergrund dieser zentralen Novellenfigur als kulturell tätiger Adlige und Mäzen vorgestellt, sondern sein kulturelles Renommee wird auch explizit dargelegt: „Graf Hoditz [...] lebte hier [...] und alles, was auf den Namen Künstler Anspruch machte, eilte von fern und nah herbei, um Wunder Roswalde's [...] kennen zu lernen." ${ }^{21}$ Der Rosswalder Garten und das Schloss werden metaphorisch als Feensitz nachzeichnet und Albert Joseph Hoditz wird die Bezeichnung des Schöpfers verliehen, wodurch der Erzähler die künstlerische Begabung dieses Grafen auffallen lässt. Hoditz wird nicht nur im Spiegel seines künstlerischen Enthusiasmus skizziert, sondern er wird auch im Lichte seiner, durch Reisen beeinflussten Ausbildung erfasst. Sein Dominium wird metaphorisch zum kulturellen Mittelpunkt stilisiert und als „Wunder Roswalde’s“22 bezeichnet. Die Pracht des Rosswalder Herrschaftsguts wird dem französischen Versailles mit der Intention gleichgestellt, auf die Phänomenalität dieses Landessitzes hinzuweisen. Obwohl die Lage des Rosswalder Dominiums als peripher zu

\footnotetext{
15 Vgl. ebenda.

16 Vgl. ebenda.

17 Vgl. ebenda.

18 Ebenda, S. 24.

19 Vgl. ebenda.

20 Ebenda, S. 25.

21 Ebenda, S. 10.

22 Ebenda.
} 
betrachten ist, wird in der Novelle ihre Bedeutung im zeitgenössischen europäischen kulturellen Panorama akzentuiert. ${ }^{23}$

Die Einsicht in das kulturelle Kolorit des Rosswalder Herrschaftsguts wird durch zahlreiche superlativische Adjektivformen gesteigert, die der Schilderung Plastizität verleihen: „Es zierte bereits der schönste Asternflor die Blumenstrassen des Palastes [...]; in den Morgenstunden versammelte munterer Hörnerschall die vornehmsten Herren und die anmutigsten Damen zur Jagd [...]; Opern und Schauspiele der überraschendsten Art währten bis tief in die Nacht. "“2 Darüber hinaus wird das Bild der kulturellen Atmosphäre am Rosswalder Hof durch die Personifikationen intensiviert, die zu ihrer suggestiven Aufwertung beitragen. ${ }^{25}$ Obwohl der auktoriale Erzähler nicht auf das Repertoire des Rosswalder Ensembles eingeht, wird die Rolle der Opern und Schauspiele im Rosswalder kulturellen Programm betont.

Der Aufenthalt des preußischen Königs im Rosswalder Schloss wird durch eine Reihe von Metaphern und wiederholt durch die Dominanz von den Attributen in der Superlativform geschildert. Sie tragen zur Akzentuierung seiner Rolle in der Geschichte des Rosswalder Dominiums bei und rücken die Gastfreundschaft des Grafen Hoditz in den Vordergrund. Das Rosswalder Fest zugunsten des Aufenthaltes Friedrich II. wird als Gipfel der kulturellen Bemühungen von Hoditz dargestellt, der sich durch eine gesellschaftlich-kulturelle Vielfalt auszeichnete: „Die Blüte der Schönheit und Künste, die vornehmsten Geschlechter und die gefeitesten Namen waren bereits in Roswalde's Mauern, und alle barg das Schloß in seinen glanzerfüllten Gemächern [...] und die herrlichsten waren bereitet, um den vornehmsten Gast zu empfangen. ${ }^{\text {" }} 6$

Der auktoriale Erzähler bietet einen Einblick in die Tätigkeit des Grafen Hoditz, durch den die Kreativität dieses Adligen hervorgehoben wird: „Die Unterhaltungen in Roswalde gingen trotz aller dieser Störungen, durch die unermüdliche Sorgfalt des Grafen Hoditz, dessen schöpferische Einbildungskraft ihn nie im Stich ließ, ihren gewohnten Gang fort. ${ }^{\text {"27 }}$ Die Intensität der kulturellen Bemühungen dieses Adligen spiegelte sich sowohl in dem zugunsten des Besuchs Friedrich II. veranstalteten Fest, als auch im literarischen Schaffen des preußischen Monarchen wider. Die Gastfreundschaft des Grafen Hoditz wird mit der Zufriedenheit Friedrichs II. quittiert, die in der Novelle Lewalds folgenden Niederschlag gefunden hat: „Friedrich war mit der Aufmerksamkeit seines Wirthes so zufrieden, daß er sein feenhaftes Roswalde in einem eigenen Gedichte besang und während seines Aufenthaltes in einer so heiteren Stimmung sich befand, die seit lange schon seine nächste Umgebung nicht an ihm wahrgenommen hatte.“"

23 Der auktoriale Erzähler thematisiert die Bedeutung des Rosswalder kulturellen Mosaiks im europäischen kulturellen Leben mit den folgenden Worten: „,...] und es genügte zu der damaligen Zeit, bei den dort veranstalteten Festlichkeiten sich auszuzeichnen, um sich einen bedeutenden Ruf in Europa zu gründen." Ebenda, S. 10.

24 Ebenda.

25 Vgl. z. B. mit dem Folgenden: „Alles athmete Freude und Lust, aber das rege Leben [...] war durch gesteigerte Thätigkeit vermehrt." Ebenda.

26 Ebenda, S. 11.

27 Ebenda, S. 93

28 Ebenda, S. 94. 
Obwohl das Fest nicht durch den Gesang Doristeas gekrönt wurde, wird ihre Handlung in die idyllische Rosswalder Gartenlandschaft sowie ins Schloss und projiziert, die die kulturellen Ambitionen des Grafen Hoditz repräsentieren. Das Novellenende bleibt den Grundsätzen des literarischen Biedermeiers treu und zielt auf die Akzentuierung der familiären und moralischen Werte ab.

\section{Schlussfolgerungen}

Die prachtvolle Einzigartigkeit der für Friedrich II. veranstalteten Feier sowie die literarischen Affinitäten der Rosswalder Schlossresidenz und deren Gartens sind in die europäische Kunst- sowie Literaturgeschichte eingegangen. Der Graf Hoditz wurde in der Novelle Lewalds zur Zeit der Hochblüte des Rosswalder kulturellen Geschehens erfasst. Dementsprechend involviert die literarische Gestaltung dieses Adligen ausschließlich positive Charakteristika, ohne zur Typisierung dieser literarischen Figur zu neigen. Das literarische Bild des kulturellen Engagements und der Kontakte sowie die Bemühungen des Grafen Hoditz um die Verbreitung der Ausbildung korrespondieren mit den Grundsätzen der Aufklärung, obwohl die Novelle thematisch im Dienste des Biedermeiers steht.

Das literarische Bild des Grafen Hoditz geht auf die Darstellung seiner kulturellen Tätigkeit ein, die auf dem Einblick in die europäische Literatur und Kunst, dem intensiven kulturellen Engagement sowie der künstlerischen Begabung dieses Adligen beruht. Seine kulturelle Tätigkeit spiegelt sich nicht nur im vorwiegend durch dramatische Aufführungen und Opern bestimmten Programm der Rosswalder Theaterbühne wider, sondern auch das landesarchitektonische Gepräge des Rosswalder Schlossgartens ist durch eine Synthese der literatur- und kunsthistorischen Ausbildung und Kreativität gekennzeichnet. Vom kulturellen Enthusiasmus dieser in der untersuchten Novelle auftauchenden Schlüsselfigur lassen sich die Bestrebungen um die Entdeckung und Ausbildung der talentierten Künstler und das mit diesen Ambitionen des Grafen zusammenhängende Mäzenatentum nicht trennen. Mit dem vielfältigen Mosaik der kulturellen Aktivitäten des Grafen Hoditz ist in der Novelle Lewalds nicht zuletzt die Nachzeichnung seines Naturells verknüpft, das sich durch Gastfreundschaft, Offenheit, Großzügigkeit, Schöpfungskraft und Wissensdrang auszeichnet.

Die Einzigartigkeit des Rosswalder Dominiums und die Umsetzung der kulturellen Vorhaben von Hoditz werden durch zahlreiche rhetorische Mittel mit der Intention wiedergegeben, die Phänomenalität dieses kulturellen Mittelpunkts an der Peripherie zu unterstreichen. Dementsprechend wird die mit dem auktorialen Erzählmodus verknüpfte Schilderung des Rosswalder kulturellen Panoramas durch die Dominanz von adjektivischen Superlativformen hervorgehoben. Die Bedeutsamkeit der Rosswalder Kunststätte wird durch die Vergleiche mit dem Versailler Hof hervorgehoben. Das kulturelle Geschehen sowie die Gartenszenerie werden durch Metaphern bzw. Personifikationen nahegebracht und akzentuieren ihr vielfältiges Gepräge.

Die untersuchte Novelle öffnet den Raum für die Schilderung eines Ausschnittes aus der reichhaltigen Geschichte des Rosswalder Herrschaftsguts. Durch die für diese 
literarische Gattung kennzeichnende Prägnanz und Kürze wird der Beitrag der kulturellen Initiativen von Hoditz an der musikalischen Karriere einer von den Sängerinnen verdeutlicht, obwohl durch seine Bestrebungen um die Ausbildung begabter Künstler später einige berühmte Schauspieler und Sängerinnen ans Licht der europäischen Kulturentwicklung gebracht wurden. Durch diesen Einblick in die Blütezeit der kulturellen Initiativen von Hoditz gewinnt die Schilderung des Rosswalder kulturellen Renommees an Glaubwürdigkeit und Brisanz, durch die gleichsam die Erforschung ihrer Resonanz in der deutschsprachigen Literatur untermauert wird.

\section{Archivalien}

Zemský archiv v Opavě/Landesarchiv Troppau (ZAO), Fond Slezské Rudoltice/Fond Rosswald (FSR), Inventarnr.: 10452, Signatur: A 26.

\section{Literatur}

FRIEDRICH II. (1849): CEuvres de Frédéric le Grand. Bd. XIII. Berlin.

LEWALD, August (1833): Novellen. 3. Teil. Hamburg.

MYŠKA, Milan (2011): Hrabě Hodic a jeho svět. Zámecká kultura ve Slezsku mezi barokem a osvícenstvím. Ostrava.

SELBMANN, Rolf (1985): Lewald, August. In: Neue Deutsche Biographie. Bd. 14. München.

TRAMPLER, Richard (1873): Der Sonderling Hoditz in Rosswald. Eine historische Skizze. In: Österreichisch-schlesisches Vereinskalender für das Jahr 1876, Jg. 2. Wien, S. 38-74.

Doc. Mgr. Iveta Zlá, Ph.D. / iveta.zla@osu.cz

Ostravská univerzita v Ostravě, Filozofická fakulta, Katedra germanistiky, Reální 5, 70100 Ostrava, CZ 